\title{
Protective effect of vitamin D3 and erythropoietin on renal ischemia/reperfusion-induced liver and kidney damage in rats
}

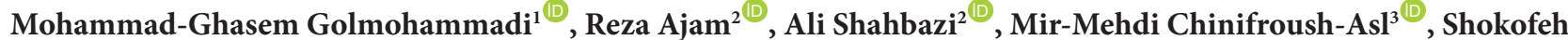 \\ Banaei $^{4^{*} \mathbb{D}}$
}

${ }^{1}$ Department of Anatomy, School of Medicine, Ardabil University of Medical Sciences, Ardabil, Iran ${ }^{2}$ General Practitioner, School of Medicine, Ardabil University of Medical Sciences, Ardabil, Iran

${ }^{3}$ Department of Pathology, School of Medicine, Ardabil University of Medical Sciences, Ardabil, Iran

${ }^{4}$ Department of Physiology, School of Medicine, Ardabil University of Medical Sciences, Ardabil, Iran

\section{A R T I C L E I N F O}

Article Type:

Original Article

\section{Article History:}

Received: 10 January 2020

Accepted: 6 April 2020

\section{Keywords:}

Erythropoietin

Ischemia-reperfusion

Vitamin D3

Kidney

Liver

\begin{abstract}
A B S T R A C T
Introduction: Renal ischemia reperfusion (IR) contributes to the development of acute renal failure (ARF). Free radicals are considered to be principal components involved in the pathophysiological alterations observed during IR. In this study, we evaluated the effects of vitamin D and erythropoietin (EPO) in IR-induced renal and liver damage.

Methods: Wistar rats were divided into five groups of 6 each. 1) The control, 2) IR, 3) VD3 (1,25-dihydroxyvitamin $\mathrm{D} 3)+\mathrm{IR}, 4) \mathrm{EPO}+\mathrm{IR}$, and 5) $\mathrm{VD} 3+\mathrm{EPO}+\mathrm{IR}$ groups. The rats were unilaterally nephrectomized and subjected to 45 minutes of renal pedicle occlusion followed by $24 \mathrm{~h}$ reperfusion. Vitamin D (10 mg/kg, IP) and EPO (1000 U/kg, IP) were administered prior to ischemia. After 24 hours reperfusion, the blood samples were collected for the determination of biochemical parameters and kidney and liver samples were taken for histological studies.

Results: Renal ischemia significantly decreased kidney and liver functions. IR significantly increased blood urea nitrogen-creatinine (BUN-Cr), glucose, total protein and liver enzyme levels and significantly decreased hemoglobin ( $\mathrm{Hb}$ ) and hematocrit (Hct) values. Histopathological findings of the IR group confirmed that there were glomerular atrophy and acute tubular necrosis in the renal tissues and lymphocyte infiltration in the liver samples. Treatment with vitamin D and EPO boosted liver and kidney functions and improved the morphological changes.

Conclusion: It seems that vitamin D or EPO administration could protect the kidney and liver damage induced by IR. Also, the combination of vitamin D and EPO may exert more beneficial effects than either agent used alone.
\end{abstract}

Implication for health policy/practice/research/medical education:

Recent investigations have shown that vitamin D3, erythropoietin or their co-administration might be used for protection against ischemia reperfusion- induced liver and kidney damages.

Please cite this paper as: Golmohammadi MG, Ajam R, Shahbazi A, Chinifroush-Asl MM, Banaei S. Protective effect of vitamin D3 and erythropoietin on renal ischemia/reperfusion-induced liver and kidney damage in rats. J Herbmed Pharmacol. 2020;9(3):293-299. doi: 10.34172/jhp.2020.37.

\section{Introduction}

Ischemia (cessation of blood flow), followed by reperfusion (re-establishment of blood flow), causes severe damage to the organs (1). Ischemia compromises the continuous supply of oxygen required by cells and tissues to maintain their physiological function. Renal ischemia is a common problem during partial nephrectomy, hydronephrosis, and renal transplantation leading to kidney dysfunction and acute renal failure (ARF). ARF has high incidence rate in hospitalized patients and the most prevalent causes are ischemia-reperfusion events. When reperfusion is established, additional kidney reperfusion damage occurs. This involves the development of oxidative stress and initiates a series of cellular events that lead to apoptotic cell death (2).

Several mechanisms contribute to the pathophysiology 
of ischemia-reperfusion injury (IRI), such as inflammatory mediators, reactive oxygen species (ROS), adenosine triphosphate (ATP) depletion and accumulation of intracellular calcium and imbalance of ionic gradients across membrane. Additionally, abnormal ion and $\mathrm{pH}$ gradients disrupt membrane transport, protein production and mitochondrial functions as well as activate $\mathrm{Ca}^{2+}$-dependent enzymatic reactions (3). As a result of respiratory chain failure, generation of excessive free radicals leads to membrane lipoperoxidation and damage of cytoskeleton and cellular organelles. Excessive generation of pro-inflammatory cytokines, ROS and decreased antioxidant defense, contributes to IR injury. Therefore, ROS scavenger, antioxidant and antiinflammatory agents have been explored to be effective in reducing ischemia-reperfusion injury $(4,5)$.

Erythropoietin (EPO) is a hypoxia-inducible hematopoietic protein, which is expressed in the kidney and is a key factor in red blood cell production. The biological effects of EPO are mediated by binding to cell surface receptor (EPOR), and the presence of functional EPOR in renal tubular epithelial and mesangial cells has pointed to a potential role for EPO in the kidney (6). EPO has several protective effects, such as anti-inflammatory, antioxidant and anti-apoptotic properties which reduce oxidative stress, improve enzymatic antioxidant activities, and scavenge free radicals. Recombinant human EPO (rHuEPO) can provide impressive protection against IR damage of several organs including the heart, liver, brain and lungs. Also, it is revealed that renal EPO levels were lowered after renal IR injury (7).

Vitamin $\mathrm{D}$ is a circulating hormone in the body indispensable for mineral homeostasis. It is transported in the circulation by the vitamin $\mathrm{D}$ binding protein. In the liver, vitamin $\mathrm{D}$ is hydroxylated on its side chain to form 25-hydroxyvitamin $\mathrm{D}$ (8). The kidney is the primary site where the active form of vitamin $\mathrm{D}$, 1,25-dihydroxyvitamin $\mathrm{D} 3\left[1,25(\mathrm{OH})_{2} \mathrm{D}_{3}\right]$ or calcitriol is produced. The classical $1,25(\mathrm{OH})_{2} \mathrm{D}_{3}$ pathway requires the nuclear vitamin D receptor. Vitamin D exhibits several physiological activities, including the regulation of serum calcium homeostasis, immune functions, and antioxidant properties. Also, it affects the antioxidant defense system, by up-regulating endogenous antioxidant enzymes and inducing the expression of the genes for proteins that protect genome (9). Vitamin D is responsible for renal protection and regulation of various physiological actions as well. Therefore, vitamin D deficiency can accelerate the progression of renal failure. As mentioned above, the biological active form of vitamin $\mathrm{D}$ is produced in the kidney by mitochondria of the proximal tubules, and it was revealed that $1,25(\mathrm{OH})_{2} \mathrm{D}_{3}$ levels were lowered after renal IR injury (10).

Therefore, ROS were indicated to contribute to the cellular and tissue damage induced by ischemia- reperfusion. The purpose of this study was to examine the effects of EPO and vitamin D or their co-administration on the reduction of injury induced by ischemia-reperfusion in a rat model of renal IR using both biochemical and histological evaluations.

\section{Material and Methods}

Animals

In this experimental study, 30 adult Wistar rats (weighing 200-250 g) were obtained from the experimental animal research center, Ardabil University of Medical Sciences. The rats were housed in a room with controlled temperature $\left(22 \pm 2^{\circ} \mathrm{C}\right)$ and humidity $(60 \pm 5 \%)$ in which a $12 / 12 \mathrm{~h}$ light-dark cycle was maintained. The rats had free access to standard water and food.

Surgery and experimental protocol

Under anesthesia $(75 \mathrm{mg} / \mathrm{kg}$ ketamine hydrochloride and $8 \mathrm{mg} / \mathrm{kg}$ xylazine, IP), each rat was placed at right flunk position. After minimal dissection under the last rib, right nephrectomy was performed and then, the incision was sutured. Then, the rat was placed at left flunk position, after minimal dissection under the last rib, the left renal pedicle (artery and vein) was exposed. It was occluded by placing an atraumatic microvascular clamp for 45 minutes to induce ischemia and then subjected to reperfusion for 24 hours. All rats had right nephrectomy and the presence of ischemia was visually confirmed by observing blanching of the kidney.

\section{Animal groups}

The rats were randomly divided into five groups of six rats in each group. The control group $(\mathrm{N}=6)$ underwent only nephrectomy without occlusion. The other groups were as follows:

- IR group

- $\quad$ VD3 (1,25-dihydroxyvitamin D3)+ IR group

- $\quad \mathrm{EPO}+\mathrm{IR}$ group

- $\quad \mathrm{VD} 3+\mathrm{EPO}+\mathrm{IR}$ group

EPO (Neorecormom, Roche, Mannheim, Germany) was administered as a $1000 \mathrm{U} / \mathrm{kg}$ single dose IP, 30 minutes before ischemia (11). Vitamin D3 (Deva, Kocaeli/ Turkey) was diluted with sterile saline and adjusted to a final concentration of $10 \mathrm{mg} / \mathrm{kg}$. VD3 was injected intraperitoneally, prior to ischemia (12).

\section{Biochemical analysis}

Blood samples, liver, and left kidney were obtained after 24 hours of reperfusion in each group. Blood samples were collected by heart puncture for measurement of biochemical analysis. The blood samples were centrifuged at approximately $4000 \mathrm{~g}$ for 10 minutes. The blood urea nitrogen (BUN) and creatinine (Cr) levels in the serum were determined to assess the renal function, using the autoanalyser (Alycon 300 USA) with original kits. 
Hematocrit $(\mathrm{Hct})$ and hemoglobin $(\mathrm{Hb})$ readings were also recorded for all rats. The $\mathrm{Hb}$ concentration in $\mathrm{g} / \mathrm{dL}$ was measured by cyanmethemoglobin method (13).

The activities of ALP (alkaline phosphatase), ALT (alanine transaminase), AST (aspartate transaminase), Bili (bilirubin), calcium, phosphorus, and serum glucose concentration were assayed using Randox assay kits (Randox laboratory limited). Serum total protein content was measured using the Biuret method. All measurements were done using a spectronic spectrophotometer (Bausch and Lomb, Rochester, NY, USA) (14,15).

Histological evaluation

Liver and renal tissue samples were fixed in $10 \%$ buffered formalin solution, dehydrated in ascending grades of alcohol and embedded in paraffin. Sections of $5 \mu \mathrm{m}$ were taken, stained with hematoxylin and eosin (H\&E), and then examined under light microscope (Olympus $\mathrm{BH}$ 2, Tokyo, Japan) in a blinded manner by pathologist. Histological changes were scored on a 4-point scale: (-) none, $(+)$ mild, $(++)$ moderate, and $(+++)$ severe damage (16).

\section{Statistical analysis}

All the data are presented as mean \pm standard deviation (SD). Evaluation of differences between groups was performed using one-way analysis of variance (ANOVA) followed by post hoc complementary test to correct for multiple comparison treatments, with SPSS 19.0 software. A $P$ value of less than 0.05 was considered statistically significant.

\section{Results}

The effect of VD3 and EPO on renal ischemia reperfusion (IR) injury was investigated in 45 minutes of renal ischemia followed by 24 hours reperfusion. Biochemical analysis results are outlined in Table 1 . Also, the results of histological evaluation are shown in Tables 2 and 3.

Effects of ischemia reperfusion

The BUN-Cr levels were significantly higher in the animals from IR group compared with those from control group $(P<0.001)$. Hct and $\mathrm{Hb}$ values in the IR group were significantly lower than those in the control group $(P<0.05$; Table 1$)$. The level of serum bilirubin in the IR group was higher than that of the control group. Also, the levels of serum total protein and glucose in the IR group were significantly higher than those of the control group $(P<0.01)$. In the IR group, the calcium level was higher and phosphorus level was lower than those of the control group. The enzymes such as AST, ALT and ALP are responsible for the proper functioning of the liver. These enzymes were significantly elevated in the IR group in comparison to control group $(P<0.01$; Table 1$)$.

Histological examination of the kidneys and liver showed that there were no histological changes in the control group (Figures 1A, 2A). In the IR group, renal injury was very obvious. There were tubular lumen dilation, cell detachment, dilated Bowman's space, glomerular atrophy, degeneration, and mononuclear cell infiltration following IR compared with the control group (Figure 1B). Also, in the IR group, the normal histological organization of the liver tissues was disrupted. There were congestion,

Table 1. Biochemical measurements after 24 hours of reperfusion

\begin{tabular}{|c|c|c|c|c|c|}
\hline & Control & IR & VD3+IR & EPO+IR & VD3+EPO+IR \\
\hline BUN (mg/dL) & $54.66 \pm 8.61$ & $139.21 \pm 96.54^{\mathrm{a}}$ & $127.51 \pm 19.35$ & $119.25 \pm 40.70$ & $84.93 \pm 12.43$ \\
\hline $\mathrm{Cr}(\mathrm{mg} / \mathrm{dL})$ & $0.68 \pm 0.06$ & $0.96 \pm 0.49$ & $0.83 \pm 0.43$ & $0.90 \pm 0.19$ & $0.81 \pm 0.12$ \\
\hline Hct(\%) & $44.01 \pm 3.38$ & $40.35 \pm 4.42^{b}$ & $46.11 \pm 3.65^{c}$ & $47.19 \pm 3.45^{c}$ & $43.33 \pm 2.15^{c}$ \\
\hline $\mathrm{Hb}(\mathrm{g} / \mathrm{dL})$ & $14.65 \pm 1.12$ & $13.45 \pm 1.46^{b}$ & $15.35 \pm 1.20^{c}$ & $15.75 \pm 1.09^{c}$ & $14.44 \pm 0.71^{c}$ \\
\hline Bili(mg/dL) & $0.15 \pm 0.04$ & $0.20 \pm 0.11$ & $0.14 \pm 0.05$ & $0.19 \pm 0.09$ & $0.13 \pm 0.04$ \\
\hline Glucose(mg/dL) & $83.83 \pm 11.05$ & $153.51 \pm 31.60^{\mathrm{a}}$ & $150.93 \pm 49.66$ & $141.20 \pm 40.93$ & $138.60 \pm 32.29$ \\
\hline Total protein(mg/dL) & $6.80 \pm 0.48$ & $7.73 \pm 0.32^{\mathrm{a}}$ & $6.86 \pm 0.38^{d}$ & $7.13 \pm 0.82^{\mathrm{d}}$ & $6.48 \pm 0.40^{d}$ \\
\hline Calcium(mg/dL) & $9.12 \pm 4$ & $9.53 \pm 1.35$ & $11.40 \pm 1.26$ & $9.41 \pm 1.33$ & $9.55 \pm 0.65$ \\
\hline Phosphorus(mg/dL) & $5.70 \pm 0.91$ & $4.03 \pm 1.41$ & $8.18 \pm 5.38$ & $4.78 \pm 1.40$ & $6.28 \pm 1.86$ \\
\hline $\operatorname{AST}(\mathrm{U} / \mathrm{L})$ & $98.16 \pm 36.79$ & $352.83 \pm 91.07^{a}$ & $204.33 \pm 45.33^{d}$ & $140.50 \pm 28.24^{d}$ & $169.66 \pm 26.91^{\circ}$ \\
\hline $\operatorname{ALT}(U / L)$ & $48.33 \pm 9.56$ & $87.50 \pm 21.85^{\mathrm{a}}$ & $69.00 \pm 6.89$ & $63.00 \pm 6.26$ & $52.16 \pm 9.10^{d}$ \\
\hline $\operatorname{ALP}(U / L)$ & $256.50 \pm 41.60$ & $314.50 \pm 119.31$ & $235.83 \pm 80.70$ & $252.50 \pm 90.63$ & $299.50 \pm 70.65$ \\
\hline
\end{tabular}

IR; Ischemia reperfusion, VD3; Vitamin D3, EPO; Erythropoietin, BUN; Blood urea nitrogen, Cr; Creatinine, Hct; Hematocrit, Hb; Hemoglobin, Bili; Bilirubin, AST; Aspartate transaminase, ALT; Alanine transaminase, ALP; Alkalin phosphatase.

a Significantly increased when compared with control group, $P<0.05$.

${ }^{\mathrm{b}}$ Significantly decreased when compared with control group, $P<0.05$.

'Significantly increased when compared with IR group, $P<0.05$.

${ }^{\mathrm{d}}$ Significantly decreased when compared with IR group, $P<0.05$. 
Table 2. Tubular and glomerular changes in the kidney after 24 hours reperfusion (H\&E)

\begin{tabular}{|c|c|c|c|c|c|c|}
\hline Groups & Glomerular atrophy & Degeneration & Acute tubular injury & Mononuclear cell infiltration & Congestion & Hyaline cast \\
\hline Control & - & - & - & - & - & - \\
\hline IR & +++ & +++ & +++ & +++ & +++ & +++ \\
\hline VD3 & - & + & + & - & + & - \\
\hline EPO & - & - & + & + & - & +++ \\
\hline VD3+EPO & - & - & - & ++ & - & - \\
\hline
\end{tabular}

At least 10 fields for each kidney slide were examined and assigned for severity of changes using scores on a scale of: (-) none, (+) mild, (++) moderate, and $(+++)$ severe damage. $(n=6$ for each group).

Table 3. Histopathological changes in the liver after $24 \mathrm{~h}$ reperfusion (H\&E)

\begin{tabular}{lccc}
\hline Groups & $\begin{array}{c}\text { Lymphocyte } \\
\text { infiltration }\end{array}$ & $\begin{array}{c}\text { Portal vein } \\
\text { congestion }\end{array}$ & $\begin{array}{c}\text { Microvesicular } \\
\text { steatosis }\end{array}$ \\
\hline Control & - & - & - \\
IR & +++ & +++ & +++ \\
VD3 & - & ++ & - \\
EPO & ++ & + & - \\
VD3+EPO & + & - & - \\
\hline
\end{tabular}

At least 10 fields for each liver slide were examined and assigned for severity of changes using scores on a scale of: $(-)$ none, $(+)$ mild, $(++)$ moderate, and $(+++)$ severe damage. ( $n=6$ for each group)

lymphocyte infiltration, triaditis, and microvesicular steatosis following IR compared with the control group (Figure 2B).

Effects of vitamin D3 on renal ischemia reperfusion Serum BUN-Cr levels in the VD3 + IR group were lower than those of the IR group. Hct and $\mathrm{Hb}$ values in the VD3 + IR group were significantly higher than those in the IR group $(P<0.001$; Table 1$)$. Serum bilirubin level in the VD3 + IR group was lower than that in the IR group. Also, the levels of serum total protein and glucose in the VD3 + IR group were lower than those of the IR group. In the VD3 + IR group, the calcium and phosphorus levels were higher than those of the IR group. The levels of AST, ALT and ALP were decreased in the VD3 + IR group in comparison to the IR group (Table 1).

Vitamin D administration resulted in marked reduction of histopathological alterations induced by IR in renal and liver tissues (Figures 1C, 2C).

\section{Effects of EPO on renal IR}

Serum BUN-Cr levels in the EPO+IR group were lower than those of the IR group. Hct and $\mathrm{Hb}$ values in the EPO+IR group were significantly higher than those in the IR group $(P<0.001$; Table 1$)$. Serum bilirubin level in the EPO + IR group was lower than that in the IR group. Also, the levels of serum total protein and glucose in the $\mathrm{EPO}+\mathrm{IR}$ group were lower than those of the IR group. In the EPO + IR group, the calcium level was lower and phosphorus level was higher than those of the IR group. The levels of AST, ALT and ALP were decreased in the
$\mathrm{EPO}+\mathrm{IR}$ group in comparison to the IR group (Table 1) In the EPO group, there were tubular hyaline cast and lymphocyte infiltration. Also moderate histopathological alterations were observed in the renal and liver tissues (Figures 1D, 2D).

Effects of vitamin D3 and EPO on renal IR

In the $\mathrm{VD} 3+\mathrm{EPO}+\mathrm{IR}$ group, the serum levels of BUN$\mathrm{Cr}$, bilirubin, total protein and glucose were lower than those of the IR group. Hct and Hb values in this group were significantly higher than those in the IR group $(P<0.05)$. Also, in this group, the calcium and phosphorus levels were higher than those of the IR group. The levels of AST, ALT and ALP were significantly decreased in the VD3+EPO+IR group in comparison to the IR group $(P<0.05$; Table 1$)$.

VD3 and EPO co-treatment severely attenuated the histopathological changes, nearly the normal structure of renal and liver tissues was preserved by VD3 and EPO pretreatment. Therefore, combination therapy appears to have beneficial effects on ischemia-reperfusion injury (Figures 1E, 2E).

\section{Discussion}

Renal IR is a common result of clinical procedures such as partial nephrectomy, and kidney transplantation. Furthermore, kidney IR injury is a leading cause of acute kidney injury, which is associated with high mortality rates. Acute kidney injury is characterized by increased vascular resistance in the kidney, a low rate of filtration through the glomeruli, and tubular necrosis. These deleterious effects have been attributed to the generation of ROS and infiltration of inflammatory cells during renal reperfusion $(17,18)$.

In the present study, renal IR injury resulted in both glomerular and tubular dysfunctions (19). IR significantly increased the serum levels of BUN and creatinine, suggesting an impaired glomerular function which were greatly reduced by VD3 or EPO treatment. It is shown that the administration of EPO before ischemia attenuated the deterioration in renal function as a result of IR injury. The administration of EPO as a single dose before the onset of ischemia has produced a significant decrease in tubular damage, which was associated with a marked amelioration of renal functional impairment as assessed by biochemical 

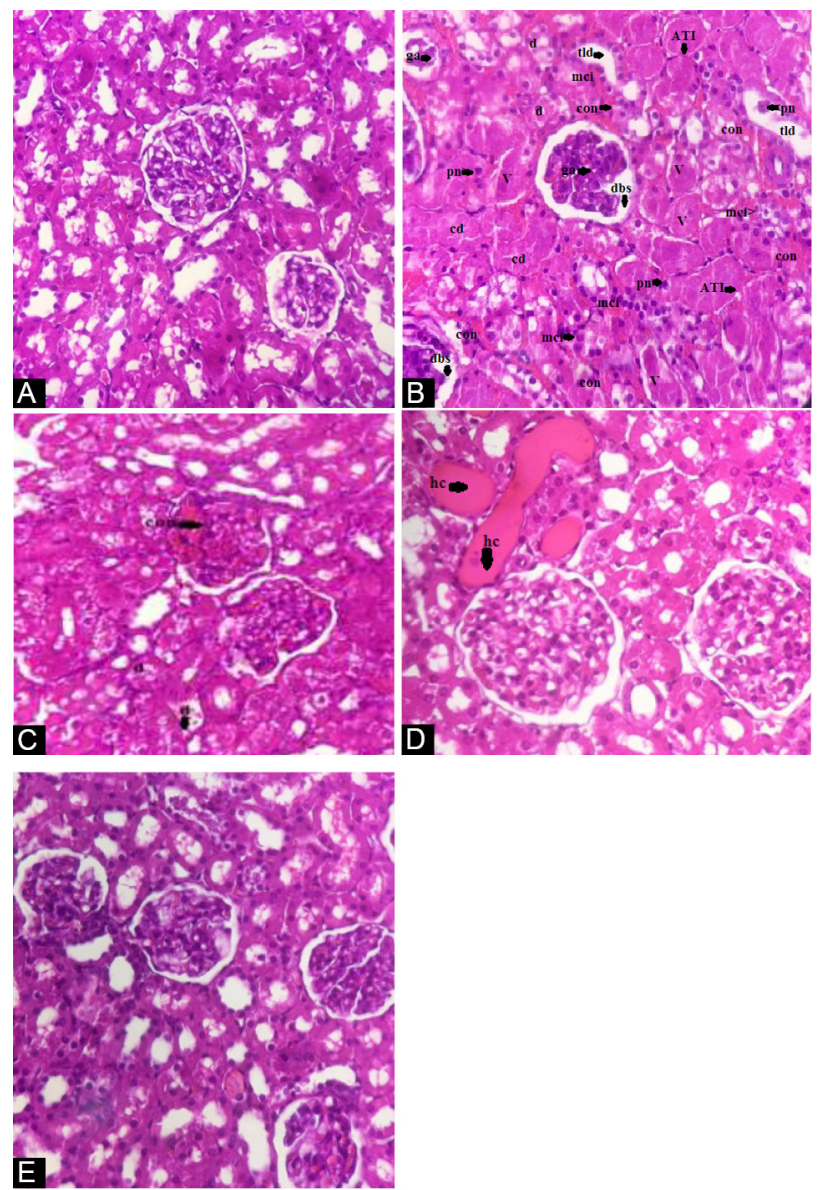

Figure 1. Histopathological evaluation of rat kidneys after $45 \mathrm{~min}$ of ischemia followed by $24 \mathrm{~h}$ of reperfusion. Kidney sections were stained by hematoxylin and eosin (H\&E) and examined by a light microscope. (A) The normal renal tissue structure in the control group. Healthy appearance of glomerular and tubular cells $(40 \times \mathrm{HE})$. (B) Tubular lumen dilation (tld), vacuolization (v), degeneration (d), cell detachment (cd), acute tubular injury (ATI), congestion (con), dilated Bowman's space (dbs), glomerular atrophy (ga), mononuclear cell infiltration (mci), and pyknotic nuclei $(\mathrm{pn})$ in the IR group $(40 \times \mathrm{HE})$. (C) The lesser degree of degeneration and congestion in the VD3 group $(40 \times \mathrm{HE})$. (D) The degree of hyaline cast in the EPO group $(40 \times \mathrm{HE})$. (E) The normal renal tissue structure in the VD3+EPO group $(40 \times \mathrm{HE})$. The arrows show the disorders on the photomicrographs.

parameters (6). It is indicated that the administration of VD3 retards deterioration of renal function and structure. There is also recent evidence that VD3 regulates glomerular filtration and renal tubular function (9). Also, we found that the serum levels of BUN and $\mathrm{Cr}$ decreased by combination of two agents (VD3+EPO), which indicates that VD3+EPO improves the recovery of renal function.

In our study, $\mathrm{Hb}$ and $\mathrm{Hct}$ values were significantly reduced by renal IR. As mentioned above, the biological active form of vitamin $\mathrm{D}$ is produced in the kidney by mitochondria of the proximal tubules, and both 1,25(OH)2 D3 and EPO levels are reduced after renal IR injury. Also, EPO is a hematopoietic growth factor, which increases the values of $\mathrm{Hb}$ and Hct. Thus, this effect of IR may be due to decrease of renal EPO level after renal IR. We explored that VD3 and EPO significantly increased
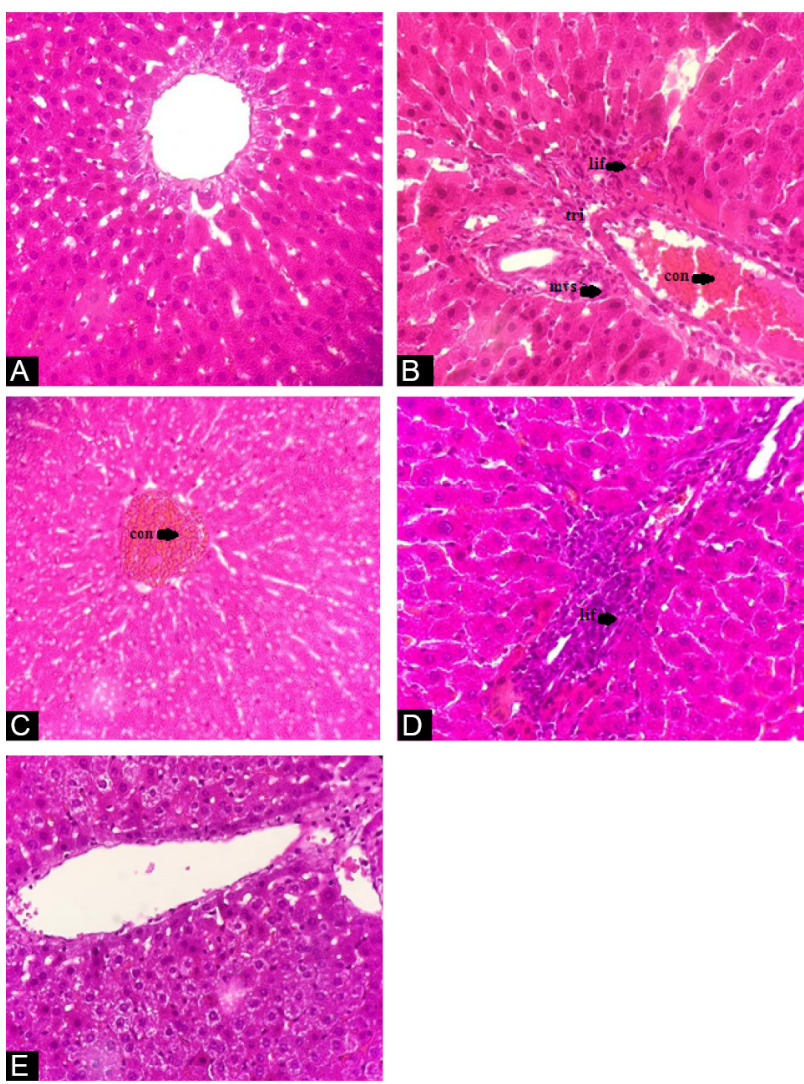

Figure 2. Histopathological evaluation of rat liver after $45 \mathrm{~min}$ of renal ischemia followed by $24 \mathrm{~h}$ of reperfusion. Liver sections were stained by hematoxylin and eosin (H\&E) and examined by a light microscope. (A) The normal liver tissue structure in the control group. (B) Congestion (con), lymphocyte infiltration (lif), triaditis (tri), and microvesicular steatosis (mvs) in the IR group $(40 \times \mathrm{HE})$. (C) Portal vein congestion (con) in the VD3 group. (D) Lymphocyte infiltration (lif) in the EPO group. (E) The normal liver tissue structure in the VD3+EPO group $(40 \times \mathrm{HE})$.

the values of $\mathrm{Hb}$ and $\mathrm{Hct}(20)$. Also, the combination of the two agents (VD3+EPO) significantly increased the values of $\mathrm{Hb}$ and $\mathrm{Hct}$.

The obtained findings demonstrated an increase in ALT, AST and ALP in the liver. Renal IR injury induces oxidative DNA damage towards the animal liver and kidney tissues and causes adverse changes in animal biochemical markers, especially total protein, glucose and serum bilirubin levels. It seems that the high oxidative power of ischemia-reperfusion is the main reason for these disturbances. Our results indicated that renal IR induced kidney and liver damages like previous studies. Moreover, ischemia- reperfusion is a common problem during organ transplantation in clinical situations and leads to organ dysfunction and delayed graft function. Also, the production of toxic metabolites during ischemia insult causes the rejection of transplanted kidney in endstage renal disease patients. Reducing the toxicity of free radicals in IR injury can be an important goal in leading research and a protective approach against IR damage. It also can improve the outcome after renal transplantation in these patients. 
Therefore, the compounds that can inhibit the toxicity of ROS will be particularly important compounds in IR injury. Vitamins, such as vitamin D, E, C, have always been proved in controlling and preventing various toxic metabolites. In this study, the effect of vitamin D and EPO was assessed on liver and renal damage mediated by renal IR injury. Vitamin D, regulates cell differentiation, cell growth and immune system and prevents neoplastic transformation. Also, it plays a major role in the secretion of insulin from pancreatic beta cells and regulation of reconstruction of bone. The most active form of vitamin $\mathrm{D}, 1,25$-dihydroxy vitamin $\mathrm{D} 3$, has a major role in calcium and phosphorous homeostasis. On the other hand, the kidney is the primary site where the active form of vitamin $\mathrm{D}$ is produced and this production is decreased by renal IR injury. Recent findings demonstrated that vitamin D had protective effects in ischemic injury, cardiovascular and renal diseases. It seems the protective mechanism of vitamin $\mathrm{D}$ in kidney diseases is regulated by the reninangiotensin system and mediated by various receptors (12).

In our study, the treatment with vitamin $\mathrm{D}$ decreased AST, ALT, and ALP in the liver. The antioxidant effect of vitamin $\mathrm{D}$ is an additional role to its calcium regulating role. Due to having a homologous structure to cholesterol, it has been proposed that this vitamin may be regarded as a potent antioxidant. The expression of several antioxidant enzymes is involved in vitamin D-mediated protection from oxidative stress. In this regard, vitamin D induces the expression of catalase, glutathione peroxidase and superoxide dismutase in several tissues as this can be a potential mechanism by which vitamin $\mathrm{D}$ can exert antioxidant defenses (12).

Furthermore, we showed that treatment with EPO and combination of two agents (VD3+EPO) reduced AST, ALT, and ALP in the liver and decreased the serum levels of bilirubin, glucose and total protein. Recent investigations have shown that EPO exerts a strong protective effect against IR injury, demonstrating anti-inflammatory and antioxidant effects (21). EPO protects insulinproducing B-cells of the pancreatic islets of Langerhans from cytokine-induced destruction in a dose-dependent manner and may promote the survival of cell through an autocrine or paracrine mechanism (22).

$\mathrm{EPO}$ is a renal cytokine that regulates hematopoiesis, specifically, it can block the normal apoptotic cycle by affecting the JAK/STAT5 pathway or the BCL2 pathway. It seems to play a protective role by shielding against ischemic, hypoxic, necrotic, and apoptotic stress. Moreover, the pancreatic islets of Langerhans transduced with EPO indicated normal insulin secretion and were protected from apoptosis. Besides, EPO is cytoprotective to islets without altering islet function, increases the insulin secretion from pancreatic beta cells and reduces the level of serum glucose (22).
In this study, histological evaluation showed that IR caused glomerular and tubular changes as shown by tubular lumen dilation, congestion, degeneration, glomerular atrophy. Renal IR also caused an increase in interstitial inflammatory infiltration. EPO treatment attenuated the histopathological changes associated with renal IR injury. Moreover, attenuating effect of EPO on the morphological changes in renal tissue caused by IR injury has been reported (1). Sezgin et al (9) reported that vitamin D had protective effects on IR-induced renal injury and the histopathological changes were reversed by VD3 treatment. Also, they proposed that vitamin $\mathrm{D}$ appears to play a cytoprotective role in the kidney insulted by IR. Supporting this proposal, we have found that VD3 has protective effects on tubular function. This cytoprotective effect of VD3 may be due to its powerful antioxidant properties. Also, VD3 and EPO combination treatment reduced the histopathological changes in renal tissue caused by IR injury. VD3+EPO severely attenuated the histopathological changes, nearly the normal renal tissue structure was preserved by VD3+EPO pretreatment. Therefore, histological evaluation indicated that combination therapy might have synergistic protective effect.

Also, renal IR caused histopathological alterations in the liver tissue including portal vein congestion, lymphocyte infiltration, and microvesicular steatosis. It seems that high oxidative stress power of renal IR injury is the main reason for these disturbances (23). On the other hand, we explored that the effect of single applications of vitamin D or EPO and the combination of the two agents attenuated the histopathological changes of the liver tissue. These protective effects of vitamin D and EPO may be due to their potent anti-inflammatory and antioxidant properties, which were documented by the reduction in histopathological alterations in the liver and renal tissues. However, the combination treatment was the most effective compared with the other treatment groups.

\section{Conclusion}

In conclusion, recent investigations have indicated that vitamin D and EPO have significant antioxidant effects. Our results indicated that administration of vitamin $\mathrm{D}$ or EPO may have beneficial effects on IR-induced liver and renal injury as showed by the reduction of the histopathological changes, liver and renal dysfunction. Also, the treatment with vitamin D and EPO decreased the glucose elevation-induced by IR. However, vitamin D and EPO combination treatment exerted more protective effects than single application of them. Therefore, combined therapy may be an alternative option for the decrease of IR-induced renal and liver injury.

\section{Authors' contributions}

MGG performed the design and statistical analysis; RA 
and AS English writing and grammar correction; MMCA histological evaluation; SB study design and biochemical analysis and manuscript writing. All authors read and approved the final manuscript.

\section{Conflict of interests}

The authors declare no conflict of interest.

\section{Ethical considerations}

The study was approved by Ardabil University of Medical Sciences Ethical Committee (Ethical code: IR.ARUMS. REC.1398.380). The study was conducted in accordance with the Guide for the Care and Use of Laboratory Animals published by the US National Institutes of Health.

\section{Funding/Support}

This study was financially supported by Ardabil University of Medical Sciences (Grant number: 1723)

\section{References}

1. Ahmadiasl N, Banaei S, Alihemmati A. Combination antioxidant effect of erythropoietin and melatonin on renal ischemia-reperfusion injury in rats. Iran J Basic Med Sci. 2013;16(12):1209-16.

2. Ahmadiasl N, Banaei S, Alihemmati A, Baradaran B, Azimian E. The anti-inflammatory effect of erythropoietin and melatonin on renal ischemia reperfusion injury in male rats. Adv Pharm Bull. 2014;4(1):49-54. doi: 10.5681/ apb.2014.008.

3. Plotnikov EY, Chupyrkina AA, Jankauskas SS, Pevzner IB, Silachev DN, Skulachev VP, et al. Mechanisms of nephroprotective effect of mitochondria-targeted antioxidants under rhabdomyolysis and ischemia/ reperfusion. Biochim Biophys Acta. 2011;1812(1):77-86. doi: 10.1016/j.bbadis.2010.09.008.

4. Hagiwara S, Koga H, Iwasaka H, Kudo K, Hasegawa A, Kusaka J, et al. ETS-GS, a new antioxidant, ameliorates renal ischemia-reperfusion injury in a rodent model. J Surg Res. 2011;171(1):226-33. doi: 10.1016/j.jss.2010.01.039.

5. Andrianova NV, Zorova LD, Babenko VA, Pevzner IB, Popkov VA, Silachev DN, et al. Rapamycin is not protective against ischemic and cisplatin-induced kidney injury. Biochemistry (Mosc). 2019 ;84(12):1502-1512. doi: 10.1134/ S0006297919120095.

6. Ahmadiasl N, Banaei S, Alihemati A, Baradaran B, Azimian E. Effect of a combined treatment with erythropoietin and melatonin on renal ischemia reperfusion injury in male rats. Clin Exp Nephrol. 2014;18(6):855-64. doi: 10.1007/s10157014-0937-6.

7. Banaei S, Ahmadiasl N, Alihemmati A. Combination anti-apoptotic effect of erythropoietin and melatonin on ischemia reperfusion-induced renal injury in rats. Acta Med Iran. 2016;54(10):624-30.

8. Sinanoglu O, Sezgin G, Ozturk G, Tuncdemir M, Guney S, Aksungar FB, et al. Melatonin with 1,25-dihydroxyvitamin D3 protects against apoptotic ischemia-reperfusion injury in the rat kidney. Ren Fail. 2012;34(8):1021-6. doi: 10.3109/0886022X.2012.700887.

9. Sezgin G, Ozturk G, Guney S, Sinanoglu O, Tuncdemir M. Protective effect of melatonin and 1,25-dihydroxyvitamin
D3 on renal ischemia-reperfusion injury in rats. Ren Fail. 2013;35(3):374-9. doi: 10.3109/0886022X.2012.760409.

10. Kim YO, Li C, Sun BK, Kim JS, Lim SW, Choi BS, et al. Preconditioning with 1,25-dihydroxyvitamin D3 protects against subsequent ischemia-reperfusion injury in the rat kidney. Nephron Exp Nephrol. 2005;100(2):e85-94.

11. Moore E, Bellomo R. Erythropoietin (EPO) in acute kidney injury. Ann Intensive Care. 2011;1(1):3. doi: 10.1186/21105820-1-3.

12. Hafez AA, Naserzadeh $\mathrm{P}$, Ashtari K, Mortazavian AM, Salimi A. Protection of manganese oxide nanoparticlesinduced liver and kidney damage by vitamin D. Regul Toxicol Pharmacol. 2018;98:240-244. doi: 10.1016/j. yrtph.2018.08.005.

13. Mahoney JJ, Vreman HJ, Stevenson DK, Van Kessel AL. Measurement of carboxyhemoglobin and total hemoglobin by five specialized spectrophotometers (CO-oximeters) in comparison with reference methods. Clin Chem. 1993;39(8):1693-700.

14. Kalantari H, Jalali M, Jalali A, Mahdavinia M, Salimi A, Juhasz B, et al. Protective effect of Cassia fistula fruit extract against bromobenzene-induced liver injury in mice. Hum Exp Toxicol. 2011;30(8):1039-44. doi: $10.1177 / 0960327110386256$.

15. Sulaiman FA, Adeyemi OS, Akanji MA, Oloyede HOB, Sulaiman AA, Olatunde A, et al. Biochemical and morphological alterations caused by silver nanoparticles in Wistar rats. J Acute Med. 2015; 5 (4): 96-102. doi:10.1016/j. jacme.2015.09.005.

16. Kiris I, Kapan S, Kilbas A, Yilmaz N, Altuntas I, Karahan N, et al. The protective effect of erythropoietin on renal injury induced by abdominal aortic-ischemia-reperfusion in rats. J Surg Res. 2008;149(2):206-13. doi: 10.1016/j.jss.2007.12.752.

17. Banaei S, Rezagholizadeh L. The role of hormones in renal disease and ischemia-reperfusion injury. Iran J Basic Med Sci. 2019;22(5):469-476. doi: 10.22038/ijbms.2019.34037.8095.

18. Mazani M, Mahmoodzadeh Y, Chinifroush Asl MM, Banaei S, Rezagholizadeh L, Mohammadnia A. Renoprotective effects of the methanolic extract of Tanacetum parthenium against carbon tetrachloride-induced renal injury in rats. Avicenna J Phytomed. 2018;8(4):370-379.

19. Alzahrani FA. Melatonin improves therapeutic potential of mesenchymal stem cells-derived exosomes against renal ischemia-reperfusion injury in rats. Am J Transl Res. 2019;11(5):2887-2907.

20. Banaei S, Ahmadiasl N, Alihemmati A. Comparison of the protective effects of erythropoietin and melatonin on renal ischemia-reperfusion injury. Trauma Mon. 2016;21(3):e23005.

21. Tasdemir S, Parlakpinar H, Vardi N, Kaya E, Acet A. Effect of endogen-exogenous melatonin and erythropoietin on dinitrobenzene sulfonic acid-induced colitis. Fundam Clin Pharmacol. 2013;27(3):299-307. doi: 10.1111/j.14728206.2011.01016.x.

22. Fenjves ES, Ochoa MS, Gay-Rabinstein C, Molano RD, Pileggi A, Mendez AJ, et al. Adenoviral gene transfer of erythropoietin confers cytoprotection to isolated pancreatic islets. Transplantation. 2004;77(1):13-8.

23. Yousefi H, Ahmadiasl N, Salimnejad R, Bagheri E, Roshangar L, Alihemmati A. Effects of renal ischemia-reperfusion on biochemical factors and histopathological alterations in the liver of male rats. Physiol Pharmacol. 2019;23(1):44-50. 